\title{
Glutamatergic Biomarkers for Cocaine Addiction: A Longitudinal Study Using MR Spectroscopy and mGluR5 PET in Self-Administering Rats
}

\author{
Bart de Laat $^{1,2}$, Akila Weerasekera ${ }^{1,3}$, Gil Leurquin-Sterk ${ }^{2}$, Guy Bormans ${ }^{1,4}$, Uwe Himmelreich ${ }^{1,3}$, Cindy Casteels ${ }^{1,2}$, \\ and Koen Van Laere ${ }^{1,2}$ \\ ${ }^{I}$ Molecular Small Animal Imaging Center (MoSAIC), KU Leuven-University of Leuven, Leuven, Belgium; ${ }^{2}$ Division of Nuclear \\ Medicine, Department of Imaging and Pathology, KU Leuven-University of Leuven/University Hospital Leuven, Leuven, Belgium; \\ ${ }^{3}$ Biomedical MRI Unit, Department of Imaging and Pathology, KU Leuven-University of Leuven, Leuven, Belgium; and ${ }^{4}$ Laboratory \\ for Radiopharmacy, Department of Pharmaceutical and Pharmacological Sciences, KU Leuven-University of Leuven, Leuven, \\ Belgium
}

Cocaine addiction is a disorder that still lacks diagnostic biomarkers or effective pharmacotherapy. We present findings on a rat model of cocaine self-administration that was followed up longitudinally using the metabotropic glutamate receptor type 5 (mGluR5) tracer ${ }^{18} \mathrm{~F}-3-$ fluoro-5-[(pyridin-3-yl)ethynyl]benzonitrile ( $\left.{ }^{18} \mathrm{~F}-\mathrm{FPEB}\right) \mathrm{PET}$, proton MR spectroscopy ( $\left.{ }^{1} \mathrm{H}-\mathrm{MRS}\right)$, and behavioral tests. Methods: Forty-two Wistar rats were scanned with ${ }^{18} \mathrm{~F}$-FPEB PET and ${ }^{1} \mathrm{H}-\mathrm{MRS}$ before and after sucrose or intravenous cocaine self-administration, during withdrawal, and during relapse. All animals performed a rodent lowa Gambling Task (rIGT) at baseline to evaluate decision making. Baseline values were used in a mixed model to assess associations with later cocaine use, and follow-up measurements were compared with the values before drug exposure. Results: Preexposure rIGT scores were significantly related to both cocaine and sucrose use during the drug-exposure phase. However, only cocaine self-administration induced a decrease in ${ }^{18} \mathrm{~F}$-FPEB binding. This decrease was most pronounced bilaterally in the hippocampus, where mGluR5 availability correlated with the amount of cocaine used during relapse. Compared with the sucrose group, a larger decrease was observed in the hippocampo-prefrontal cortex pathway. Preexposure glutamate and glycine concentrations in the prefrontal cortex were significantly associated with cocaine use during the drug-exposure phase. Moreover, prefrontal glutamate exhibited a distinct, reversible decrease when animals had access to cocaine but not sucrose. Conclusion: Baseline values of prefrontal glutamate and glycine are associated with future cocaine use. Furthermore, baseline rIGT scores are associated with both sucrose and cocaine. Finally, both glutamate concentration and mGluR5 availability decrease during exposure to cocaine.

Key Words: cocaine; ${ }^{18} \mathrm{~F}-\mathrm{FPEB} ;{ }^{1} \mathrm{H}-\mathrm{MRS}$; rodent lowa gambling task; prefrontal cortex

J Nucl Med 2018; 59:952-959

DOI: 10.2967/jnumed.117.202614

Received Sep. 22, 2017; revision accepted Dec. 15, 2017.

For correspondence or reprints contact: Koen Van Laere, KU Leuven, Herestraat 49-bus 7003 59, 3000 Leuven, Belgium.

E-mail: koen.vanlaere@uzleuven.be

Published online Mar. 1, 2018.

COPYRIGHT (C 2018 by the Society of Nuclear Medicine and Molecular Imaging.
$\mathbf{T}$ o date, therapeutic options for drug-use disorders remain limited despite intensive research to unravel their complex neurobiology (1). This complexity is partly due to the dynamic changes during different phases of these disorders. For example, neurologic control mechanisms that modulate drug-induced release of dopamine become progressively more prominent when the brain is repeatedly exposed. The increased role of these inhibitory mechanisms can ultimately result in phenotypical traits associated with cocaine abuse, such as poor decision making and impulsivity. For example, cocaine users are known to perform worse on the Iowa Gambling Task, a widely used measure of decision making (2). These controlling mechanisms are thought to be in place mainly as a result of the glutamatergic system, with the prefrontal cortex as its predominant enforcer (3). In this context, the extrasynaptic metabotropic glutamate receptors have gained considerable interest (4). These receptors, in particular metabotropic glutamate receptor types 2 and 5 (mGluR5), have a modulatory role that is disturbed in several psychiatric disorders, including addiction (5). In cocaine addiction, mGluR5 knockout mice have been used to show that the absence of this receptor influences conditioned locomotor activity and the signaling cascade involved in the propensity to self-administer $(6,7)$.

Several drugs have been designed to modulate the glutamatergic system, aiming at an antiaddictive effect (8). However, most were plagued by toxicity and side effects or lacked convincing results in clinical trials (9). It has been hypothesized that the disappointing results are due to a lack of insight into the dynamics of addiction and to the absence of suitable biomarkers to guide pharmacopsychologic therapy (10). Because of these issues, there has risen a demand to elucidate the role of the glutamatergic system during different phases of addiction, such as drug exposure, withdrawal, and relapse (11).

In this context, we conducted a longitudinal experiment on a cocaine self-administration rat model. This protocol commenced before the exposure to cocaine and followed each animal through its initial drug exposure, but also through withdrawal and relapse. In every phase, animals were characterized using ${ }^{18} \mathrm{~F}-3$-fluoro-5[(pyridin-3-yl)ethynyl]benzonitrile $\left({ }^{18} \mathrm{~F}-\mathrm{FPEB}\right)$ PET for mGluR5 imaging, as well as noninvasive proton MR spectroscopy $\left({ }^{1} \mathrm{H}-\mathrm{MRS}\right)$ for glutamate and glycine concentration measurement. Additionally, 
at baseline a behavioral test for decision making, namely the rodent Iowa Gambling Task (rIGT; Med Associates), was performed. This information was used to study whether there are baseline markers associated with future drug use. We also assessed where and how these potential biomarkers changed during the different phases of the addictive process.

\section{MATERIALS AND METHODS}

\section{Animals}

Cocaine-exposure experiments were performed in duplicate, within a total of 36 animals originating from these 2 identical and independent experiments $8 \mathrm{wk}$ apart. In both experiments, 18 adult (110$115 \mathrm{~d}$ old) male Wistar rats (Janvier Laboratories) weighing on average $( \pm \mathrm{SD}) 500 \pm 40 \mathrm{~g}$ were used. Additionally, a matched control group was used to control for cocaine-independent effects. Because sucrose induces homogeneous self-administration behavior, we anticipated a low level of variance in the control group. Therefore, a smaller number of animals, that is, 6 , were included in this group. On arrival at our facility, all rats were housed individually under an inversed 12-h light-dark cycle. With free access to water and $20 \pm 1 \mathrm{~g}$ of food (Ssniff) per day, a stable body weight was maintained. At least $7 \mathrm{~d}$ before the self-administration sessions, the rats had a polyurethane 22-gauge catheter (Instech Laboratories) inserted through the femoral vein in the cranial direction for $7 \mathrm{~cm}$. A vascular access button was mounted between the shoulder blades using a polyethylene terephthalate mesh. The research protocol was approved by the Animal Ethics Committees of the University of Leuven (P156/2013) and was performed in accordance with the guidelines of the European Ethics Committee (decree 86/609/EEC).

\section{Self-Administration}

Nine experimental test chambers (Med Associates) equipped with a solid floor and 2 levers were used for self-administration experiments. The rats were trained to use the levers with sucrose pellets $(45 \mathrm{mg}$, TestDiet) as a reward in 10 training sessions until 100 responses were achieved within $1 \mathrm{~h}$. After the baseline week, the rats could selfadminister cocaine hydrochloride $(0.3 \mathrm{mg} / \mathrm{kg} / \mathrm{infusion})$ by pressing the active lever 1 time for $3 \mathrm{~h}$ per day for 14 consecutive days. Cocaine, dissolved in $0.9 \% \mathrm{NaCl}$, was administered intravenously via a pump infusing $0.05 \mathrm{~mL} /$ infusion over $2 \mathrm{~s}$. Each administration resulted in a 15-s time-out to prevent lethal doses. This drug-exposure phase was followed by a forced withdrawal period and a subsequent regained access for $7 \mathrm{~d}$, mimicking a relapse (Fig. 1). Catheter patency was tested using a $4 \mathrm{mg} / \mathrm{kg}$ dose of propofol (Propovet; Abott) before the first selfadministration session and whenever animals showed a more than $20 \%$ decrease in lever presses within $3 \mathrm{~d}$. Control animals followed the same experimental phases but continued to receive sucrose pellets as a reward.

\section{rIGT}

Decision making was assessed with the rIGT during the baseline phase and after catheter implantation. In this test, animals can poke their nose into any of 5 holes, each with its own reward-punishment profile (profile $=$ chance of success, reward size [number of pellets], time-out duration [s]), as described by Zeeb et al. (12). More specifically, the largest reward size could be obtained by solely choosing the (profile $=0.8,2,10$ ) response hole, whereas the riskier choice of the (profile $=0.4,4,40$ ) option resulted in the smallest reward size. The outcome, decision-making score, was calculated according to the following formula:

$$
\text { Score }=\frac{\# \text { pellets rewarded }}{\# \text { nose-pokes }}-2
$$

with the " 2 " being the score of optimal decision making.

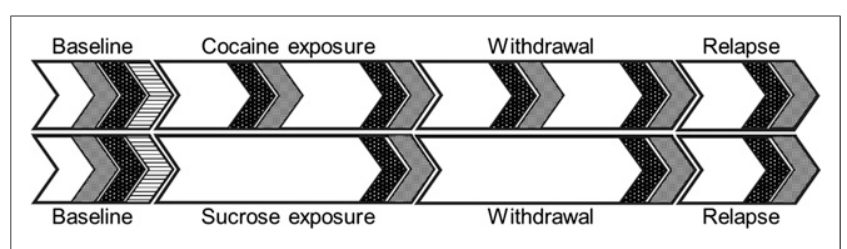

FIGURE 1. Schematic overview of experimental design for cocaine group (top) and control group (bottom). From left to right, each set of patterns represents rIGT, ${ }^{18} \mathrm{~F}-\mathrm{FPEB}$ PET, and ${ }^{1} \mathrm{H}-\mathrm{MRS}$.

\section{Small-Animal PET Imaging}

PET imaging was always performed at approximately the same time during the dark cycle on a lutetium oxyorthosilicate detector-based smallanimal tomograph (Focus 220; Siemens Medical Solutions). During imaging, the rats were kept under $2.0 \%$ isoflurane anesthesia, and their body temperature was maintained with a heating pad. Scans were obtained at baseline $(n=33)$, during drug-exposure weeks 1 and 2 ( $n=24$ and 25, respectively), during withdrawal weeks 1 and 2 ( $n=15$ and $=21$, respectively), and during relapse $(n=22)$, as a function of the available amount of radiotracer produced and its specific activity. (The control animals were scanned only at baseline, during drug-exposure week 2 , during withdrawal week 2 , and during relapse.) On average, $18.5 \pm 3.6 \mathrm{MBq}$ (specific activity range, $80-301 \mathrm{GBq} / \mu \mathrm{mol}$; mass dose range, $1.03-5.64 \mu \mathrm{g}$ ) were injected into the tail vein using an infusion needle set. For each 60-min dynamic scan, 3 animals were placed in the PET scanner. They simultaneously received an ${ }^{18} \mathrm{~F}-\mathrm{FPEB}$ injection, upon which the emission scan and subsequent ${ }^{57} \mathrm{Co}$-attenuation scan were started.

Nondisplaceable binding $\left(\mathrm{BP}_{\mathrm{ND}}\right)$ parametric images were calculated, upon spatial normalization in the Paxinos stereotactic space, with the previously validated simplified reference tissue model, using the cerebellum as a reference region (13). A volume-of-interest-based analysis was performed to assess the value of baseline measurements. In contrast, changes in ${ }^{18} \mathrm{~F}-\mathrm{FPEB} \mathrm{BP}_{\mathrm{ND}}$ between different phases were assessed voxelwise with Statistical Parametric Mapping, version 12 (SPM) (14). For the SPM analysis, data were analyzed with a flexible factorial design (Tables 1 and 2) and a cluster extent threshold of 200. Significance thresholds were set at a familywise error-corrected $P$ value of less than 0.00001 for the comparison between the cocaine and sucrose groups. For the longitudinal evaluation of the cocaine group, we applied a familywise error-corrected $P$ value of less than 0.005 for phases with drug exposure, whereas an uncorrected $P$ value of less than 0.0005 was used for both withdrawal weeks. In addition, a voxel-based correlation analysis between the median self-administration and ${ }^{18} \mathrm{~F}-\mathrm{FPEB} \mathrm{BP}_{\mathrm{ND}}$, or prefrontal glutamate concentration, was performed.

\section{${ }^{1}$ H-MRS}

${ }^{1} \mathrm{H}-\mathrm{MRS}$ was performed on a subset of 18 randomly selected animals from the cocaine group, which were scanned each week, as well as all 6 animals in the sucrose group. All MR images and spectra were acquired with a $9.4 \mathrm{~T}$ Biospec small-animal MR scanner (Bruker Biospin) equipped with a horizontal-bore magnet and an actively shielded gradient set of $600 \mathrm{mT} / \mathrm{m}$ using a $7-\mathrm{cm}$ linearly polarized resonator for transmitting and an actively decoupled dedicated rat surface coil for receiving (Rapid Biomedical). For the placement of ${ }^{1} \mathrm{H}$ MRS voxels, 2-dimensional T2-weighted MR images were acquired. Spectroscopy voxels were manually placed over 2 a priori-selected regions: the left nucleus accumbens $(2.5 \times 2.5 \times 2.5 \mathrm{~mm})$ medioventral to the caudate putamen, and the prefrontal cortex $(2 \times 3 \times 2.5 \mathrm{~mm})$ caudad to the rhinal fissure and craniodorsal to the corpus callosum (Supplemental Fig. 1; supplemental materials are available at 
TABLE 1

SPM Results for Clusters of Difference in mGluR5 Availability Between Cocaine and Sucrose Group

\begin{tabular}{|c|c|c|c|c|c|c|c|c|c|c|c|}
\hline \multirow[b]{2}{*}{ Parameter } & \multirow[b]{2}{*}{ Threshold $(P)$} & \multicolumn{4}{|c|}{ Cluster level } & \multicolumn{2}{|c|}{ Voxel level } & \multicolumn{3}{|c|}{ Coordinate } & \multirow[b]{2}{*}{ Anatomic structure } \\
\hline & & $P_{\text {Corr }}$ & $\mathrm{K}_{\mathrm{E}}$ & $\begin{array}{l}\Delta S \\
(\%)\end{array}$ & $\begin{array}{l}\Delta \mathrm{C} \\
(\%)\end{array}$ & $\mathrm{T}$ & $P_{\text {Corr }}$ & $x$ & $y$ & $z$ & \\
\hline \multirow[t]{5}{*}{ Baseline > DE2 } & 0.00001 (FWE) & 0.0001 & 1,631 & 3 & -14 & 7.90 & 0.0001 & 2.1 & -0.9 & 5.2 & CPU L, NAC L, AI L, OC L \\
\hline & & 0.0001 & 780 & -0 & -15 & 7.82 & 0.0001 & -2.3 & -0.8 & 5.0 & CPU R, NAC R \\
\hline & & 0.0001 & 397 & -3 & -16 & 6.85 & 0.0001 & -5.5 & -1.0 & 6.5 & AI L, OC L \\
\hline & & 0.0001 & 439 & -2 & -15 & 6.69 & 0.0001 & -5.2 & -6.8 & 6.7 & Hippocampus R $(\mathrm{CA} 1, \mathrm{~S})$ \\
\hline & & 0.0001 & 234 & 0 & -13 & 6.34 & 0.0001 & -5.2 & -5.8 & 7.2 & Hippocampus L (CA1, S) \\
\hline \multirow[t]{2}{*}{ Baseline $>$ W2 } & 0.00001 (FWE) & 0.0001 & 502 & 0 & -15 & 7.67 & 0.0001 & -2.3 & -0.8 & 5.0 & CPU L, NAC L \\
\hline & & 0.0001 & 701 & 5 & -14 & 7.65 & 0.0001 & 2.1 & -0.5 & 4.7 & CPU R, NAC R \\
\hline \multirow[t]{4}{*}{ Baseline $>$ relapse } & 0.00001 (FWE) & 0.0001 & 2,478 & -0 & -24 & 9.67 & 0.0001 & -2.3 & -0.8 & 5.0 & CPU L, NAC L, AI L, OC L \\
\hline & & 0.0001 & 2,892 & 0 & -13 & 9.34 & 0.0001 & 2.1 & -0.7 & 5.1 & CPU R, NAC R, AI R, OC R \\
\hline & & 0.0001 & 387 & -5 & -15 & 6.89 & 0.0001 & 5.2 & -6.8 & 6.7 & Hippocampus R (CA1, S) \\
\hline & & 0.0001 & 387 & -7 & -13 & 6.69 & 0.0001 & -6.0 & -6.0 & 6.0 & Hippocampus L (CA1, S, DG) \\
\hline
\end{tabular}

DE2 = drug-exposure week 2; W2 = withdrawal week 2; FWE = familywise error-corrected; $\mathrm{K}_{\mathrm{E}}=$ cluster extent; $\Delta \mathrm{S}=$ percentage change between respective phases for sucrose group; $\Delta \mathrm{C}=$ percentage change between respective phases for cocaine group; $\mathrm{T}=\mathrm{T}$-score value; $\mathrm{CPU}=$ striatum; $\mathrm{NAC}=$ nucleus accumbens; $\mathrm{Al}=$ agranular insular cortex; $\mathrm{OC}=$ orbital cortex; $\mathrm{S}=$ subiculum; $\mathrm{DG}=\mathrm{dentate}$ gyrus.

http://jnm.snmjournals.org). Localized Fastmap shimming was performed before the PRESS sequence (320 averages; echo time/repetition time, $20 \mathrm{~ms} / 1.8 \mathrm{~s}$; acquisition duration, $9 \mathrm{~min} 38 \mathrm{~s}$ ). Analyses were performed with jMRUI, version 5.2, on total creatine (creatine plus phosphocreatine), $\gamma$-aminobutyrate, glucose, glutamate, glutamine, glycine, lactate, $N$-acetylaspartate, and taurine.

\section{Statistical Analysis}

All statistical analyses were performed using SAS JMP Pro, version 12.1 (SAS Institute Inc.). By design, the reported data of animals exposed to cocaine were pooled from 2 independent experiments to minimize the number of false-positive results. Moreover, effects were withheld when significant for the pooled data and either significant in both experiments separately or significant in one experiment and consistent in the other (i.e., the estimate of a significant effect in one experiment included the estimate of the other experiment within its $95 \%$ confidence interval). Therefore, no additional multiple-test comparison was applied to avoid false-negatives. This approach was not adopted for the reported SPM results because of the nature of the SPM output.

The ability of baseline measurements to explain variability in drug use during the drug-exposure phase was assessed with linear mixed models, including a random effect per animal and animal $\times$ day. To visualize these results, we divided the animals into 3 groups based on the baseline value of the variable of interest such that each group included approximately one third of the total number of animals. Self-administration results were then plotted for these 3 groups. Differences between phases were evaluated with a Kruskal-Wallis test,

TABLE 2

Longitudinal Analysis of Effect of Cocaine Self-Administration on mGluR5 Availability

\begin{tabular}{|c|c|c|c|c|c|c|c|c|c|c|}
\hline \multirow[b]{2}{*}{ Parameter } & \multirow[b]{2}{*}{ Threshold $(P)$} & \multicolumn{3}{|c|}{ Cluster level } & \multicolumn{2}{|c|}{ Voxel level } & \multicolumn{3}{|c|}{ Coordinate } & \multirow[b]{2}{*}{ Anatomic structure } \\
\hline & & $P_{\text {Corr }}$ & $\mathrm{K}_{\mathrm{E}}$ & Decrease (\%) & $\mathrm{T}$ & $P_{\text {Corr }}$ & $x$ & $y$ & $z$ & \\
\hline Baseline $>$ DE1 & 0.005 (FWE) & 0.0001 & 1,470 & $37 \pm 6$ & 5.95 & 0.01 & 3.2 & -6.2 & 6.6 & Hippocampus R (DG) \\
\hline \multirow[t]{2}{*}{ Baseline > DE2 } & 0.005 (FWE) & 0.0003 & 533 & $20 \pm 8$ & 6.90 & 0.0001 & -0.4 & -5.4 & 2.4 & Prefrontal cortex (FAC, PLC) \\
\hline & & 0.0001 & 20,986 & $25 \pm 8$ & 6.68 & 0.0001 & 2.2 & -4.8 & 5.6 & Hippocampus BL, CPU (S, DG) \\
\hline \multirow[t]{3}{*}{ Baseline $>$ relapse } & 0.005 (FWE) & 0.0001 & 14,223 & $19 \pm 7$ & 9.50 & 0.0001 & 3.8 & -6.4 & 6.8 & Hippocampus R (DG) \\
\hline & & 0.0003 & 539 & $12 \pm 7$ & 9.04 & 0.0001 & -0.2 & -5.8 & 2.4 & Prefrontal cortex (FAC, PLC) \\
\hline & & 0.0001 & 4,108 & $14 \pm 8$ & 8.35 & 0.0001 & -6.0 & -5.2 & 7.2 & Hippocampus L (CA1) \\
\hline Baseline > W1 & 0.0005 (Unc) & 0.003 & 2,330 & $19 \pm 6$ & 4.18 & 0.02 & 0.6 & -1.2 & 4.4 & Hippocampus BL (FoHC) \\
\hline Baseline > W2 & 0.0005 (Unc) & 0.002 & 2,866 & $14 \pm 5$ & 4.62 & 0.004 & -0.4 & -0.4 & 2.0 & Cingulate cortex (A2) \\
\hline
\end{tabular}

$\mathrm{DE} 1 / 2=$ drug-exposure week 1/2; W1/2 = withdrawal week 1/2; FWE = familywise error-corrected; Unc = uncorrected; $\mathrm{K}_{\mathrm{E}}=$ cluster extent; $\mathrm{T}=\mathrm{t}$-score value; $\mathrm{DG}=$ dentate gyrus; $\mathrm{FAC}=$ frontal association cortex; PLC = prelimbic cortex; $\mathrm{S}=\mathrm{subiculum}$; FoHC $=$ fimbriae of hippocampus; $\mathrm{A} 2$ = region $\mathrm{A} 2$ of cingulate cortex. 


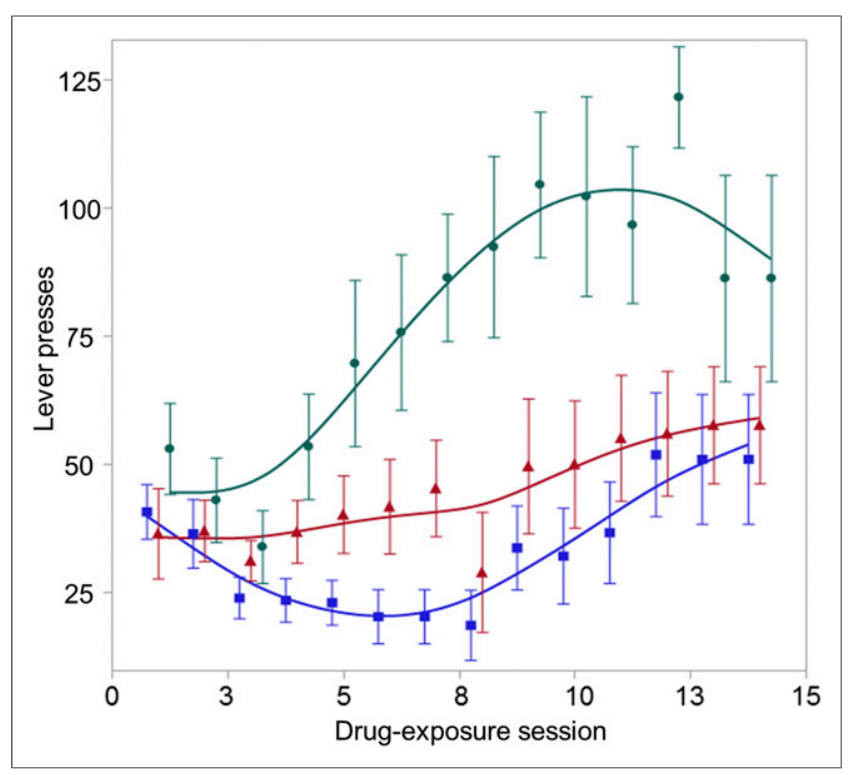

FIGURE 2. Number of lever presses during the self-administration phase. Cocaine was associated with preexposure score of decision making in rIGT. Animals were grouped according to low (blue, 0-0.15), average (red, 0.15-0.35), and high (green, 0.35-0.60) levels of decision-making score, with each category containing one third of observations.

which, if significant, was followed by a Wilcoxon signed-rank comparison. Significance was defined at the $95 \%$ confidence level. The reported values are mean $\pm \mathrm{SE}$ of the mean.

\section{RESULTS}

\section{Self-Administration}

All catheters remained patent during the experiment. Animals readily self-administered cocaine during the first and second drugexposure weeks (median number of lever presses per $3 \mathrm{~h}, 34$ and 60, respectively; interquartile range, 17-53 and 19-100, respectively). Moreover, compared with the first and second drug-exposure weeks, the relapse week showed a significant increase in cocaine intake (median, 89; interquartile range, 57-116; average, $25 \pm 2[z=9.53$, $P<0.0001])$. Sucrose intake by the control group showed a different profile, with significantly higher intake during the first

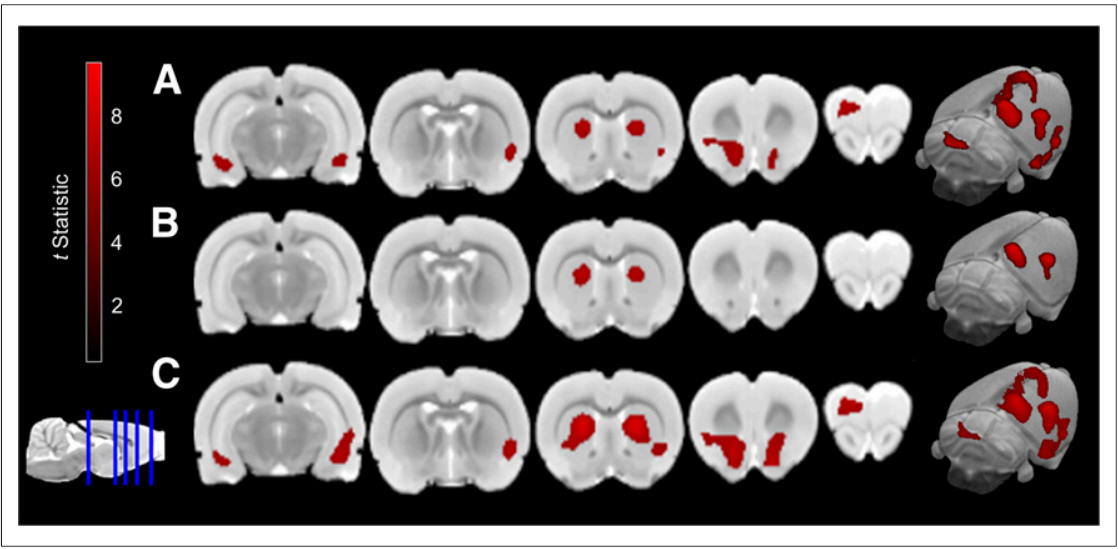

FIGURE 3. Significant SPM clusters $(P<0.00001$, familywise error-corrected) locate significant differences between rats self-administering sucrose or cocaine during drug exposure $(A)$, withdrawal (B), and relapse (C). self-administration week (median, 134; interquartile range, 103$180[z=5.55, P<0.0001])$ but no difference between the second self-administration and relapse weeks (median, 89 and 88, respectively; interquartile range, 74-123 and 69-104, respectively). Median inactive lever presses remained low throughout both the first and the second drug-exposure weeks (median, 1 and 0.5, respectively; interquartile range, $0-4$ and $0-4$, respectively), as well as during the relapse week (median, 1; interquartile range, $0-4$ ).

\section{rIGT}

The rats performed the rIGT actively, with on average $164 \pm 10$ responses in $30 \mathrm{~min}$. The mean decision-making score was $0.09 \pm$ 0.31 . The rats thus obtained $50 \pm 3$ rewards consisting of $98 \pm 6$ pellets in total. Mixed-model analysis of the baseline decisionmaking score showed a significant association with the increase in lever presses during the drug-exposure phase $\left(F_{3,78}=9.19, P=\right.$ 0.001) (Fig. 2). Indeed, an increase in decision-making score with 1 SE (0.31) increased the predicted number of lever presses by $36 \pm 8$. Interestingly, a positive association was also found in the sucrose group $\left(F_{2,6}=15.71, P=0.005\right)$, with an increase of $66 \pm$ 26 in the predicted number of lever presses for the same increase in decision-making score.

\section{Small-Animal PET Imaging}

Baseline volume-of-interest-based $\mathrm{BP}_{\mathrm{ND}}$ was used in a mixed model to assess for a potential association with substance use. However, significant results were not found for either the cocaine group or the sucrose group.

Compared with sucrose self-administration, cocaine induced a more pronounced decrease between baseline and the drug-exposure phase bilaterally in the nucleus accumbens, agranular insular cortex, and orbital cortex. Additionally, the cornu ammonis subfield 1 (CA1) and subiculum regions of the hippocampi showed a significantly larger bilateral decrease (Table 1). During withdrawal, these differences became insignificant, whereas during relapse the same regions again significantly differed between the two groups (Fig. 3). In all phases, a significantly lower $\mathrm{BP}_{\mathrm{ND}}$ for the sucrose group was found bilaterally in the striatum. Because this difference was already present at baseline, it was not evaluated in the light of this comparison.

Voxel-based analysis of the cocaine group demonstrated decreased ${ }^{18} \mathrm{~F}$-FPEB $\mathrm{BP}_{\mathrm{ND}}$ bilaterally in the hippocampus during the second drug-exposure week and during the relapse phase, as compared with baseline (Table 2). A smaller cluster of decrease was observed during the first and second withdrawal weeks (Fig. 4). During the first week of drug exposure, FPEB $\mathrm{BP}_{\mathrm{ND}}$ decreased only in the right hippocampus. Another region that showed a decrease in mGluR5 $\mathrm{BP}_{\mathrm{ND}}$ in comparison to baseline was the prefrontal cortex during the second drug-exposure week and during relapse. Lastly, a bilateral decrease of $14 \% \pm 5 \%$ was observed in the cingulate cortex during the second week of withdrawal.

Voxel-based correlation analysis showed a positive correlation $(z=3.55, P<$ $0.001)$ between the median number of lever presses for cocaine, but not sucrose, 


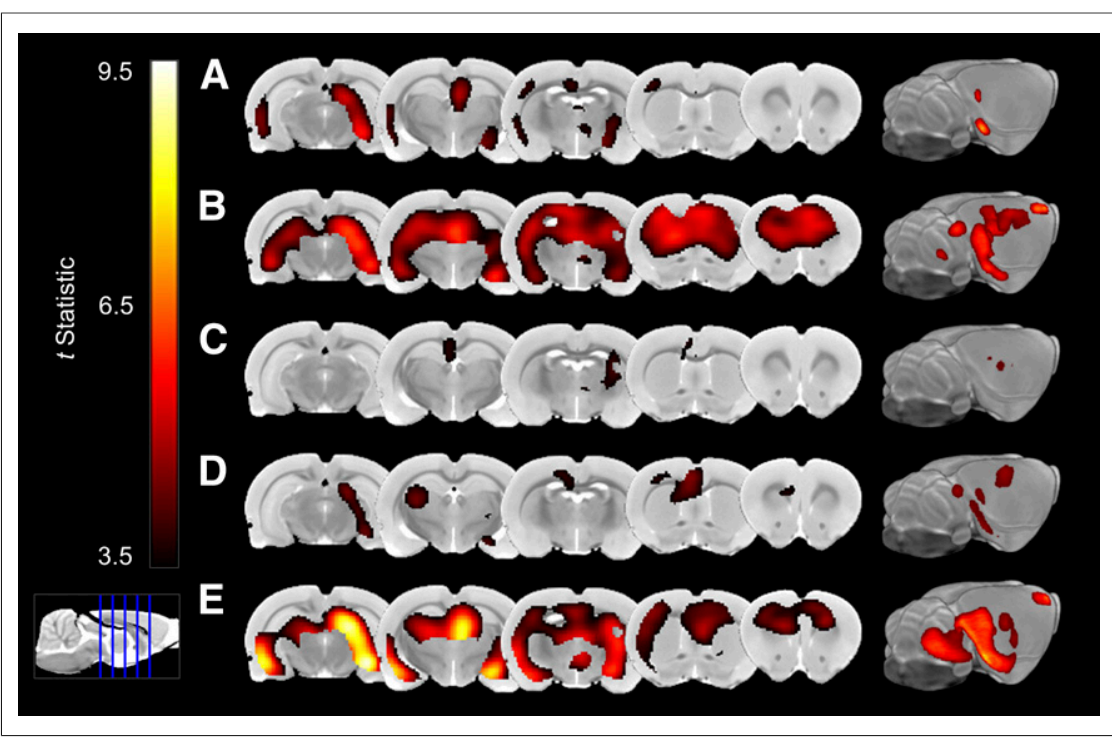

FIGURE 4. Significant SPM clusters $\left(P<0.0005\right.$, uncorrected) locate decrease in mGluR5 BP ${ }_{\mathrm{ND}}$ of cocaine group during drug-exposure week 1 (A), drug-exposure week $2(B)$, withdrawal week 1 $(C)$, withdrawal week $2(D)$, and relapse (E). Most important decreases are bilaterally in hippocampus during weeks of drug exposure. Normalization can be observed during both withdrawal weeks $(C$ and $D)$.

and ${ }^{18} \mathrm{~F}-\mathrm{FPEB} \mathrm{BP}_{\mathrm{ND}}$ in the left and right subicula of the hippocampus (respectively: cluster extent, 281 and 234; $x=-3.2$ and $3.2 ; y=8.0$ and $8.2 ; z=2.8$ and $3.2 ; P_{\text {Cluster Level }}=$ $<0.0001$ and $<0.0001)$. Classification of animals based on ${ }^{18} \mathrm{~F}-\mathrm{FPEB} \mathrm{BP}_{\mathrm{ND}}$ in this cluster suggested that this effect was most important during the relapse phase (Fig. 5).

\section{${ }^{1} \mathrm{H}-\mathrm{MRS}$}

All measured metabolites were in line with previously reported ranges for healthy rats (15). Interestingly, mixed models showed that prefrontal glutamate $\left(F_{2,36}=8.98, P<0.001\right)$ and glycine $\left(F_{2,36}=17.41, P<0.001\right)$ had a significant association with cocaine use during the drug-exposure phase (Fig. 6). On the one hand, an animal with a $1 \mathrm{mmol} / \mathrm{L}$ higher prefrontal glutamate concentration was related to, on average, $14.9 \pm 4.1$ more lever presses per session. On the other hand, an increase of $1 \mathrm{mmol} / \mathrm{L}$ in prefrontal glycine was related to an overall increase of $13.6 \pm 2.6$ lever presses. The prefrontal glutamate concentration was the only metabolite for which a significant effect of cocaine exposure was found. In particular, a decrease was observed when animals had access to cocaine during the first week of drug exposure $(1.46 \pm 0.53 \mathrm{mmol} / \mathrm{L}, z=2.74, P=$ $0.006)$ and during the relapse phase $(1.37$ $\pm 0.54 \mathrm{mmol} / \mathrm{L}, z=2.54, P=0.011$ ), compared with baseline. The first and second withdrawal weeks were also found to differ significantly from the same 2 phases - that is, the first week of drug exposure $(0.93 \pm 0.56, z=2.15, P<0.031$, and $1.01 \pm 0.65, z=2.46, P<0.013$, respectively) and the relapse phase $(0.85 \pm$ $0.56, z=1.96, P=0.049$, and $0.93 \pm$ $0.66, z=2.32, P=0.020$, respectively) (Fig. 7). No significant differences in any metabolites were found among the different phases, nor were there any significant associations with baseline measurements for the nucleus accumbens voxel and the sucrose group.

A possible relation among the reported significant baseline measurements was investigated using a nonparametric correlation analysis. No significant correlation was found between the rIGT and prefrontal glutamate or glycine concentrations. However, between the latter two, a significant correlation was found $(\rho=$ $0.77 ; P<0.0001$ ). Finally, since prefrontal concentrations of glutamate showed significant changes between the different phases, we also investigated the relationship between glutamate and mGluR5 $\mathrm{BP}_{\mathrm{ND}}$ in the prefrontal cortex, as measured with ${ }^{18} \mathrm{~F}$ FPEB PET. However, no significant association was found with the voxel-by-voxel analysis.

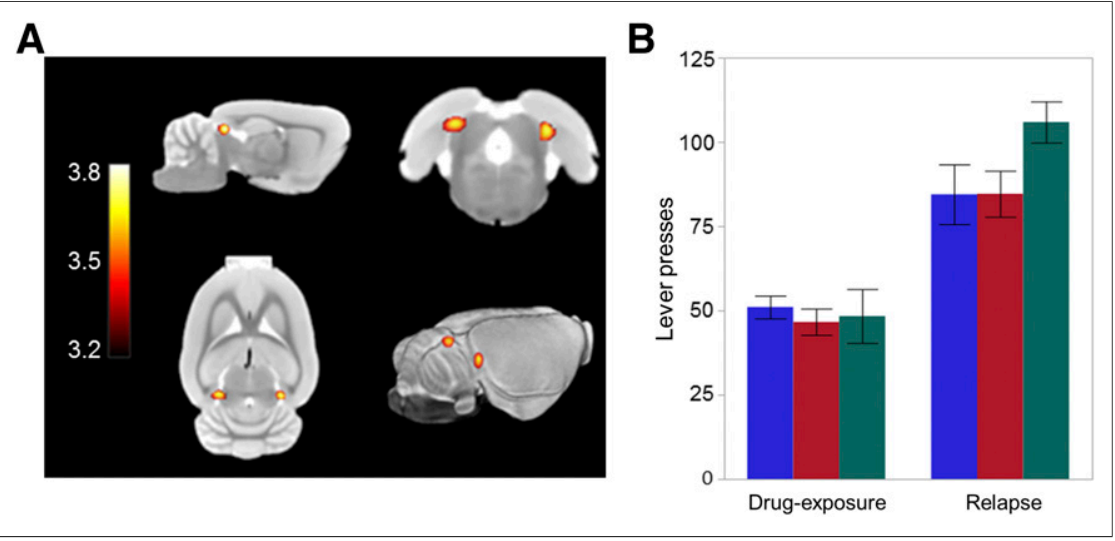

FIGURE 5. (A) Clusters of ${ }^{18} \mathrm{~F}-\mathrm{FPEB}$ binding that positively correlated with quantity of cocaine use in left and right subiculum. (B) Classification of rats based on ${ }^{18} \mathrm{~F}-\mathrm{FPEB} \mathrm{BP}_{\mathrm{ND}}$ in this cluster suggests that this association was most apparent during relapse phase. Observations are categorized by low (blue, 1.1-2.5), average (red, 2.5-3.2), or high (green, 3.3-4.4) ${ }^{18} \mathrm{~F}-\mathrm{FPEB}$ binding potential, with each group containing approximately one third of all observations. Error bars represent SEM.

\section{DISCUSSION}

In a longitudinal rat model of cocaine self-administration, we showed that baseline measurements of the rIGT, prefrontal glycine, and glutamate were associated with future cocaine use as expressed by the number of lever presses. Furthermore, mimicking of withdrawal and relapse after the initial drug exposure induced a distinct cocaine-dependent pattern in prefrontal glutamate concentration. A similar pattern was found in mGluR5 availability, explicitly for the hippocampus, where it was associated with the level of cocaine intake during the drug-exposure and relapse phases. Compared with sucrose self-administration, bilateral decreases were found in the hippocampus, nucleus accumbens, insular cortex, and orbital cortex during drugexposure phases. 


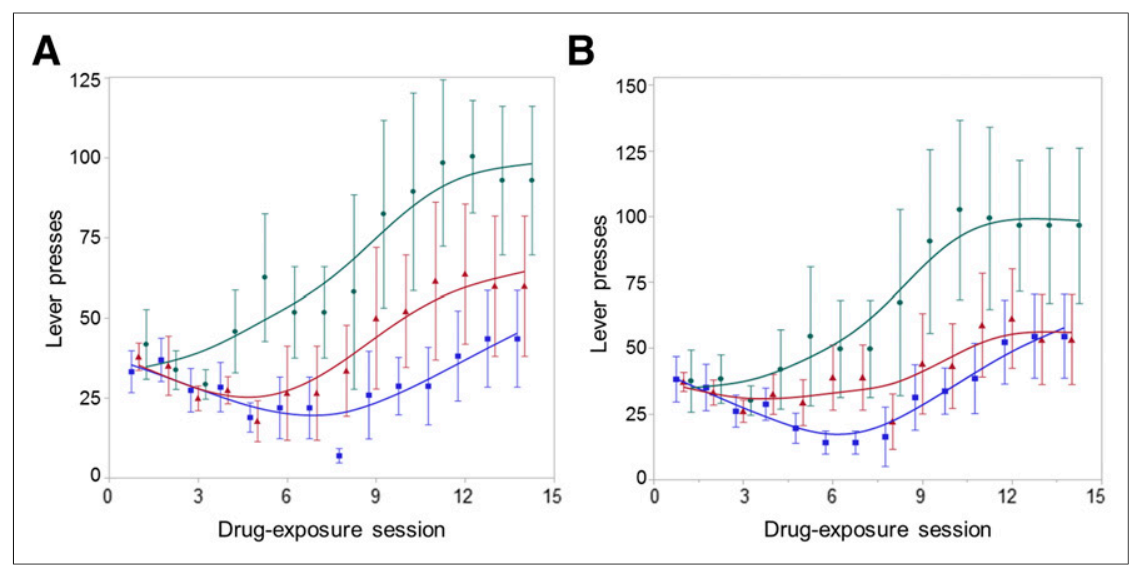

FIGURE 6. (A) Number of lever presses during the self-administration phase. Cocaine was associated with level of prefrontal glycine concentration ( $\mathrm{mmol} / \mathrm{L}$ ). For visualization purposes, rats are categorized by their prefrontal glycine concentration in low (blue, 0.20-0.45), average (red, 0.45-0.55), and high (green, 0.55-1.00) groups. Each group contained one third of all observations. (B) Cocaine use during drug-exposure phase was associated with prefrontal glutamate concentration. Rats are categorized by their prefrontal glutamate concentration in low (blue, 4.3-5.5), average (red, 5.5-6.8), and high (green, 7.2-13.5) groups, with one third of observations in each group. Error bars represent SEM.

Patients with substance-use disorders typically show impaired decision-making abilities, as they experience greater difficulty in evaluating the negative consequences of a choice (16). A longstanding question in addiction research is whether this character trait is preexisting or induced by the drug use and resulting addiction (17). Clinical studies have not provided definitive answers, because most were performed only after addiction had been established. We here show that rats with poor decision-making skills, of which specific aspects were assessed with the rIGT, had higher future intake of either sugar or cocaine (12). This finding might indicate an inverse relation between decision making and reward salience that transcends reward type (18).

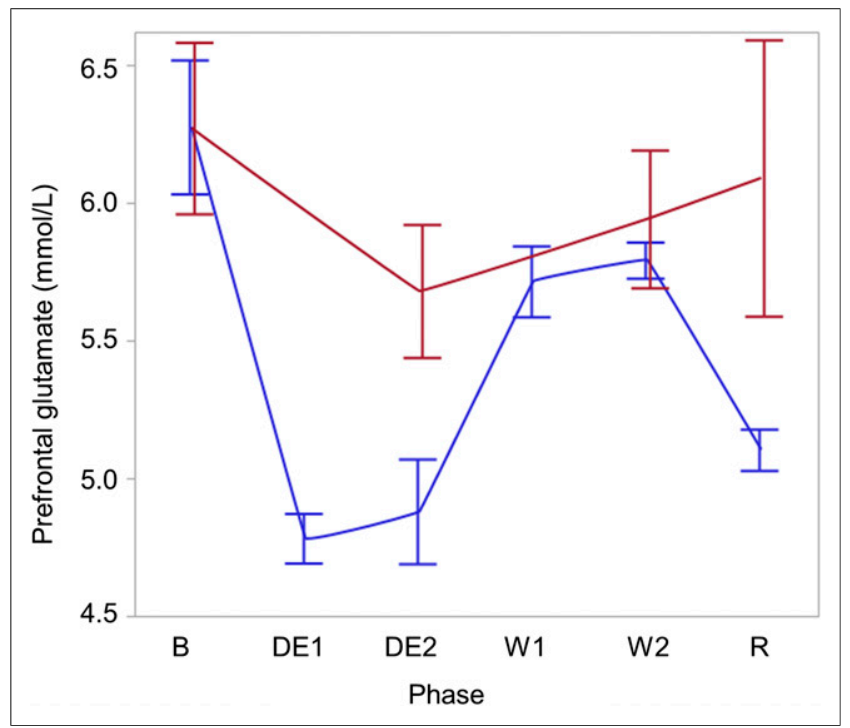

FIGURE 7. Prefrontal glutamate levels as measured with in vivo ${ }^{1} \mathrm{H}-\mathrm{MRS}$ show strong, but reversible, effect of exposure to cocaine (blue) but not to sucrose (red). $\mathrm{B}=$ baseline; DE1 = drug-exposure week 1 ; DE2 = drugexposure week $2 ; \mathrm{R}=$ regained access; $\mathrm{W} 1=$ withdrawal week $1 ; \mathrm{W} 2=$ withdrawal week 2 .
We report that, compared with sucrose, cocaine self-administration induced a significant decrease in mGluR5 availability in the brain regions involved in the hippocampo-prefrontal cortex pathway in the rat (19). This pathway originates in the CA1 and subiculum and is projected to the orbitofrontal cortex, and both sites have projections toward the nucleus accumbens. This pathway has been associated with several memory processes, including goal-oriented reward learning (20). The robust difference in mGluR5 availability in these regions between sucrose and cocaine selfadministration is remarkable and could be relevant to the ongoing debate on whether food rewards should be considered on a par with addictive substances (21). Although sucrose can be an incentive as strong as, or stronger than, cocaine, sucrose does not directly interfere with the dopaminergic system (22). Hence, one could hypothesize that the observed neurobiologic changes are due to unnaturally high synaptic dopamine levels elicited by cocaine rather than to the inherently pleasurable effects of both.

To our knowledge, this was the first study to assess mGluR5 availability in all phases of addiction. We found a decrease in mGluR5 availability after exposure to cocaine, particularly in the hippocampus, although other regions, such as the prefrontal cortex, were also implicated. This finding is in line with literature reports showing decreased mGluR5 binding in cocainedependent patients (23). Withdrawal from cocaine induced normalization of mGluR5 availability, although localized smaller decreases remained. This normalization is similar to that observed for prefrontal glutamate concentration and indicates a general downregulation of the glutamatergic system during drug exposure.

The cocaine-induced decreases were most explicit in the hippocampus - a finding that can be considered a corroboration of the hypothesis that addiction is, in large part, a pathologically strong learning process (24). Our data here suggest that the subiculum plays a specific role in this pathway, as we showed that mGluR5 densities in this region correlate with a rat's cocaine intake. The suggestion has already been made that the subiculum is involved in the formation of drug-associated memories, as lesions in this region reduce cocaine use in rats (25). This suggestion is in line with the existing hypothesis that addictive behavior arising from the center of the nucleus accumbens is heavily influenced by glutamatergic input from the subiculum (26). Therefore, mGluR5 in the subiculum might be involved in the formation of drug-associated memories, which later induce craving.

In the prefrontal cortex, which is one of the main effectors of the mesolimbic reward system, lower glutamate levels have been measured with ${ }^{1} \mathrm{H}-\mathrm{MRS}$ in human chronic users of cocaine (27). Lower extracellular basal glutamate levels have also been reported in rats after methamphetamine self-administration (28). Here, we confirm this decrease in extracellular basal glutamate levels in rats exposed to cocaine, as measured with ${ }^{1} \mathrm{H}-\mathrm{MRS}$, and show that normalization occurs during withdrawal. Our findings 
complement the data of Hermann et al., who showed that drug exposure leads to an increase in prefrontal glutamate lasting up to $60 \mathrm{~h}$ after exposure, followed by a progressive decrease to a level lower than that at baseline (29). Additionally, in humans a similar normalization is observed during alcohol abstinence (30). Indeed, Meshul et al. reported a significant decrease after $2 \mathrm{~d}$ of withdrawal that was no longer present after $14 \mathrm{~d}(31)$. However, crosssectional results alone can be misleading. For example, based on O'Neill et al., the decrease in glutamate could be considered a direct effect of withdrawal, whereas we show here that the glutamate decrease is a remnant of earlier exposure to cocaine (32). This finding emphasizes the need for longitudinal experiments in addiction research to study the temporal dynamics of biologic processes.

Glycine is an obligatory coagonist of glutamate at the $\mathrm{N}$-methylD-aspartate receptor (33), which plays an important role in the dopamine-elevating properties of drugs (34). Indeed, several rat studies have shown that antagonists of glycine can reduce the addictive properties of cocaine (35). However, to our knowledge, we are the first to report that glycine levels before drug exposure can help explain future cocaine use in rats. A clinical trial with the glycine transporter 1 inhibitor Org 25935 was performed on patients with alcohol dependence but was abandoned when evidence of efficacy was lacking (36). However, because no complete doseresponse study was performed in that trial, its true value is difficult to evaluate. In schizophrenia, in which dysregulation of the glutamatergic system is also believed to be fundamental, glycine has been found to be correlated with the severity of symptoms (37). Therefore, we believe glycine could be an interesting target deserving future studies into the pathophysiology and therapy of cocaine addiction.

\section{CONCLUSION}

We report the temporal dynamics of several important mediators of cocaine self-administration in rats before and after drug exposure. Specifically, prefrontal glycine and glutamate are found to be interesting biomarkers of vulnerability for cocaine use. Furthermore, the influence of cocaine on mGluR5 availability and prefrontal glutamate concentrations is different from the influence of sucrose. Finally, poor decision making in rats is associated with increased future cocaine self-administration. We hope that our study can provide a reference for longitudinal setups and can guide the interpretation of cross-sectional studies.

\section{DISCLOSURE}

This study was funded by grant FWO/G.0548.06 from Research Foundation Flanders (FWO), grant SB.131432 from the Flemish Agency for Innovation by Science and Technology, and grant 316679 TRANSACT from the FP7 Marie Curie project. Koen Van Laere is senior clinical research fellow for the FWO and received grant FWO/G.0548.06 for this work. Bart de Laat received a personal scholarship from the Flemish Agency for Innovation by Science and Technology. No other potential conflict of interest relevant to this article was reported.

\section{ACKNOWLEDGMENTS}

We thank Tinne Buelens and Ann Van Santvoort for providing excellent technical assistance, and we thank the radiopharmacy team UZ Leuven for producing the tracer.

\section{REFERENCES}

1. Soyka M, Mutschler J. Treatment-refractory substance use disorder: focus on alcohol, opioids, and cocaine. Prog Neuropsychopharmacol Biol Psychiatry. 2016;70:148-161

2. Verdejo-Garcia A, Benbrook A, Funderburk F, David P, Cadet JL, Bolla KI. The differential relationship between cocaine use and marijuana use on decisionmaking performance over repeat testing with the Iowa Gambling Task. Drug Alcohol Depend. 2007;90:2-11.

3. Koob GF, Volkow ND. Neurobiology of addiction: a neurocircuitry analysis. Lancet Psychiatry. 2016;3:760-773.

4. Anwyl R. Metabotropic glutamate receptor-dependent long-term potentiation. Neuropharmacology. 2009;56:735-740.

5. Kalivas PW. The glutamate homeostasis hypothesis of addiction. Nat Rev Neurosci. 2009;10:561-572.

6. Bird MK, Reid CA, Chen F, Tan HO, Petrou S, Lawrence AJ. Cocaine-mediated synaptic potentiation is absent in VTA neurons from mGlu5-deficient mice. Int $J$ Neuropsychopharmacol. 2010;13:133-141.

7. Chiamulera C, Epping-Jordan MP, Zocchi A, et al. Reinforcing and locomotor stimulant effects of cocaine are absent in mGluR5 null mutant mice. Nat Neurosci. 2001;4:873-874.

8. Schmidt HD, Pierce RC. Cocaine-induced neuroadaptations in glutamate transmission: potential therapeutic targets for craving and addiction. Ann N Y Acad Sci. 2010;1187:35-75.

9. Tomek SE, Lacrosse AL, Nemirovsky NE, Olive MF. NMDA receptor modulators in the treatment of drug addiction. Pharmaceuticals (Basel). 2013;6: 251-268.

10. Garrison KA, Potenza MN. Neuroimaging and biomarkers in addiction treatment. Curr Psychiatry Rep. 2014;16:513.

11. Cosgrove KP. A need for longitudinal studies in the addiction field. Biol Psychiatry. 2016;80:174-175.

12. Zeeb FD, Robbins TW, Winstanley CA. Serotonergic and dopaminergic modulation of gambling behavior as assessed using a novel rat gambling task. Neuropsychopharmacology. 2009;34:2329-2343.

13. de Laat B, Leurquin-Sterk G, Celen S, et al. Preclinical evaluation and quantification of ${ }^{18} \mathrm{~F}$-FPEB as a radioligand for PET imaging of the metabotropic glutamate receptor 5. J Nucl Med. 2015;56:1954-1959.

14. Statistical Parametric Mapping [computer program]. Version 12. London, U.K.: Wellcome Trust Centre for Neuroimaging; 2012.

15. Pfeuffer J, Tkac I, Provencher SW, Gruetter R. Toward an in vivo neurochemical profile: quantification of 18 metabolites in short-echo-time ${ }^{1} \mathrm{H}$ NMR spectra of the rat brain. J Magn Reson. 1999;141:104-120.

16. Paulus MP. Decision-making dysfunctions in psychiatry: altered homeostatic processing. Science. 2007;318:602-606.

17. Grant JE, Chamberlain SR. Impulsive action and impulsive choice across substance and behavioral addictions: cause or consequence? Addict Behav. 2014;39: 1632-1639.

18. Dichter GS, Damiano CA, Allen JA. Reward circuitry dysfunction in psychiatric and neurodevelopmental disorders and genetic syndromes: animal models and clinical findings. J Neurodev Disord. 2012;4:19.

19. Thierry AM, Gioanni Y, Dégénétais E, Glowinski J. Hippocamp-prefrontal cortex pathway: anatomical and electrophysiological characteristics. Hippocampus. 2000;10:411-419.

20. Godsil BP, Kiss JP, Spedding M, Jay TM. The hippocampal-prefrontal pathway: the weak link in psychiatric disorders? Eur Neuropsychopharmacol. 2013;23: 1165-1181.

21. DiLeone RJ, Taylor JR, Picciotto MR. The drive to eat: comparisons and distinctions between mechanisms of food reward and drug addiction. Nat Neurosci. 2012;15:1330-1335.

22. Cantin L, Lenoir M, Augier E, et al. Cocaine is low on the value ladder of rats: possible evidence for resilience to addiction. PLoS One. 2010;5: e11592.

23. Milella MS, Marengo L, Larcher K, et al. Limbic system mGluR5 availability in cocaine dependent subjects: a high-resolution PET $\left[{ }^{11} \mathrm{C}\right] \mathrm{ABP} 688$ study. Neuroimage. 2014;98:195-202.

24. Berridge KC, Robinson TE, Aldridge JW. Dissecting components of reward: 'liking', 'wanting', and learning. Curr Opin Pharmacol. 2009;9:65-73.

25. Caine SB, Humby T, Robbins TW, Everitt BJ. Behavioral effects of psychomotor stimulants in rats with dorsal or ventral subiculum lesions: locomotion, cocaine self-administration, and prepulse inhibition of startle. Behav Neurosci. 2001;115: 880-894.

26. Everitt BJ, Parkinson JA, Olmstead MC, Arroyo M, Robledo P, Robbins TW. Associative processes in addiction and reward: the role of amygdala-ventral striatal subsystems. Ann N Y Acad Sci. 1999;877:412-438. 
27. Yang S, Salmeron BJ, Ross TJ, Xi ZX, Stein EA, Yang Y. Lower glutamate levels in rostral anterior cingulate of chronic cocaine users: a ${ }^{1} \mathrm{H}-\mathrm{MRS}$ study using TEaveraged PRESS at 3T with an optimized quantification strategy. Psychiatry Res. 2009;174:171-176.

28. Parsegian A, See RE. Dysregulation of dopamine and glutamate release in the prefrontal cortex and nucleus accumbens following methamphetamine selfadministration and during reinstatement in rats. Neuropsychopharmacology. 2014;39:811-822.

29. Hermann D, Weber-Fahr W, Sartorius A, et al. Translational magnetic resonance spectroscopy reveals excessive central glutamate levels during alcohol withdrawal in humans and rats. Biol Psychiatry. 2012;71:10151021.

30. Umhau JC, Momenan R, Schwandt ML, et al. Effect of acamprosate on magnetic resonance spectroscopy measures of central glutamate in detoxified alcohol-dependent individuals: a randomized controlled experimental medicine study. Arch Gen Psychiatry. 2010;67:10691077.

31. Meshul CK, Noguchi K, Emre N, Ellison G. Cocaine induced changes in glutamate and GABA immunolabeling within rat habenula and nucleus accumbens. Synapse. 1998;30:211-220.
32. O’Neill J, Tobias MC, Hudkins M, London ED. Glutamatergic neurometabolites during early abstinence from chronic methamphetamine abuse. Int $J$ Neuropsychopharmacol. 2014;18:pyu059.

33. Turecek R, Trussel LO. Presynaptic glycine receptors enhance transmitter release at a mammalian central synapse. Nature. 2001;411:587-590.

34. Chau P, Soderpalm B, Ericson M. The mGluR5 antagonist MPEP elevates accumbal dopamine and glycine levels: interaction with strychnine-sensitive glycine receptors. Addict Biol. 2011;16:591-599.

35. Zhou SJ, Xue LF, Wang XY, et al. NMDA receptor glycine modulatory site in the ventral tegmental area regulates the acquisition, retrieval, and reconsolidation of cocaine reward memory. Psychopharmacology (Berl). 2012;221: 79-89.

36. de Bejczy A, Nations KR, Szegedi A, Schoemaker J, Ruwe F, Soderpalm B. Efficacy and safety of the glycine transporter-1 inhibitor org 25935 for the prevention of relapse in alcohol-dependent patients: a randomized, double-blind, placebo-controlled trial. Alcohol Clin Exp Res. 2014;38:2427-2435.

37. Hons J, Zirko R, Ulrychova M, Cermakova E, Doubek P, Libiger J. Glycine serum level in schizophrenia: relation to negative symptoms. Psychiatry Res. 2010;176:103-108. 